\title{
Research on Non-destructive Comprehensive Detection and Grading of Poultry Eggs Based on Intelligent Robot
}

\author{
Shucai Wang ${ }^{1, *}$, Jinxiu Cheng ${ }^{2}$, and Youxian Wen ${ }^{1}$ \\ ${ }^{1}$ Department of Engineering and Technology, Huazhong Agricultural University, \\ Wuhan, Hubei Province, P.R. China 430070, \\ Tel.: +86-13387580932; Fax: +86-27-87285346 \\ wsc01@mail.hzau.edu.cn \\ ${ }^{2}$ Department of Foreign Languages, Huazhong Agricultural University, \\ Wuhan, Hubei Province, P.R. China 430070
}

\begin{abstract}
This study presents a light-duty automation system for the automatization of detecting and grading poultry eggs, namely SIRDGE, short for System of Intelligent Robot Detecting and Grading Eggs. This system combines crack detection, inner quality detection and eggs conveying in detecting and grading, which are all performed by a joint-robot to fully automate egg detection and gradation. The hardware components and software structure of the system are presented. Necessary explanation of the main hardware and the software is made, which includes video image capturing and camera calibration, image processing and feature extraction, motion control and route planning of the robot, MCU control for the vacuum sucker, knocking sound processing and crack detection, color image processing and inner quality detection, etc. Property testing of SIRDGE has been done in the research.
\end{abstract}

Keywords: poultry eggs, detecting, grading, intelligent robot.

\section{Introduction}

Selling and deep processing of each egg after automatic cleaning, detecting, grading and coding enable consumers to learn about such information as the production date, brand and quality grade of each fresh egg so as to value the eggs according to quality. Moreover, it can prevent cross infection in production process so as to fulfill the traceability of the products. In other words, it not only increases the producers' profit but also ensures the consumers' interests.

In the countries with highly automatized technology such as the US, Japan and the Netherlands, processing facilities of fresh eggs include: pneumatic-sucking collecting and conveying device, cleaning and disinfection machine, coating drier, classifying and packing machine, and strikes code machine. These facilities process eggs respectively without human contact and ensure full automatization, high precision and non-destruction in processing, grading and packing. For example, FPS MOBA full

\footnotetext{
${ }^{*}$ Corresponding author.
} 
automatic classifying and packing machine by Holland Hot-Cheers Ind. Inc. can be used along with dirty eggs detection system, crack detection system, inner-blood spot and eggshell color detection system, and ultraviolet radiation sterilization system. In the event of the production demand, a system with cleaning, drying, oil coating and reassuring function can be outfitted. The products can be outfitted with full automatic material feeder as well as central collecting and conveying system connected with the henhouse.

The general methods for poultry eggs automatic detection are machine vision and acoustic features of eggs. In general, machine vision is for cracks or inner-quality detection while acoustic features of eggs for crack detection.

Whether for the export of fresh eggs or for deep egg processing, it is necessary to clean, disinfect, detect and grade eggs. At present, most enterprises in China are still employing manual work in fulfilling these tasks; as a result, there is a remarkable gap between China and developed countries both in processing quality and in efficiency.

The whole set of oversea mechatronic device for poultry eggs detection is too expensive for the small and medium-scaled enterprises in the area of eggs production and processing to afford. Under such circumstances the author uses modern computer technology achievements (machine vision, intelligent robot, etc.) to excogitate a light-duty system of intelligent robot detecting and grading eggs (SIRDGE for short), which is cheap, agile, flexible and hence fit for the small and medium-scaled enterprises. Under the guidance of machine vision, the robot can judge the position and directions of each egg, snatch and knock at eggs automatically, detect cracks by knocking sound signals, identify and grade the freshness and sizes of eggs by the image features of eggs under transmission light (backlight), and finally put the eggs in corresponding grades according to the graded signal.

\section{The Structure and Principles of SIRDGE}

SIRDGE is composed of location system (including scene video image capturing, processing and feature extraction of eggs location), conveying system (including robot motion control, manipulator and vacuum sucker system), crack recognition system (including sound producing, data acquisition and processing), and inner-quality detection system (including illumination, color image capturing and image processing).

The workflow of the system is illustrated in Fig 1. A scene camera captures the population image of eggs. Through image processing, the central coordinates and maximum axis direction of each egg are obtained. Thus joint angular displacement of the robot is solved by coordinate transformation and robot kinematic of the data. With a vacuum sucker the manipulator moves to the center of an egg, sends signals to MCU controller for the vacuum sucker, starts the vacuum suction's gas path, and then sucks the egg. According to the planned motion track, the robot carries the egg to the top of the knocking device. A microphone acquires and sends the knocking sound on the eggshell to DSP for processing, i.e. diagnosing the egg's cracks by a corresponding recognition model, the result of which is sent to a robot motion controller. If the egg is cracked, the egg will be put in the eggcrate for cracked eggs. Otherwise, the manipulator will move to the color camera and obtain the color image of the egg in the backlight. Through image processing the freshness of the egg is identified 


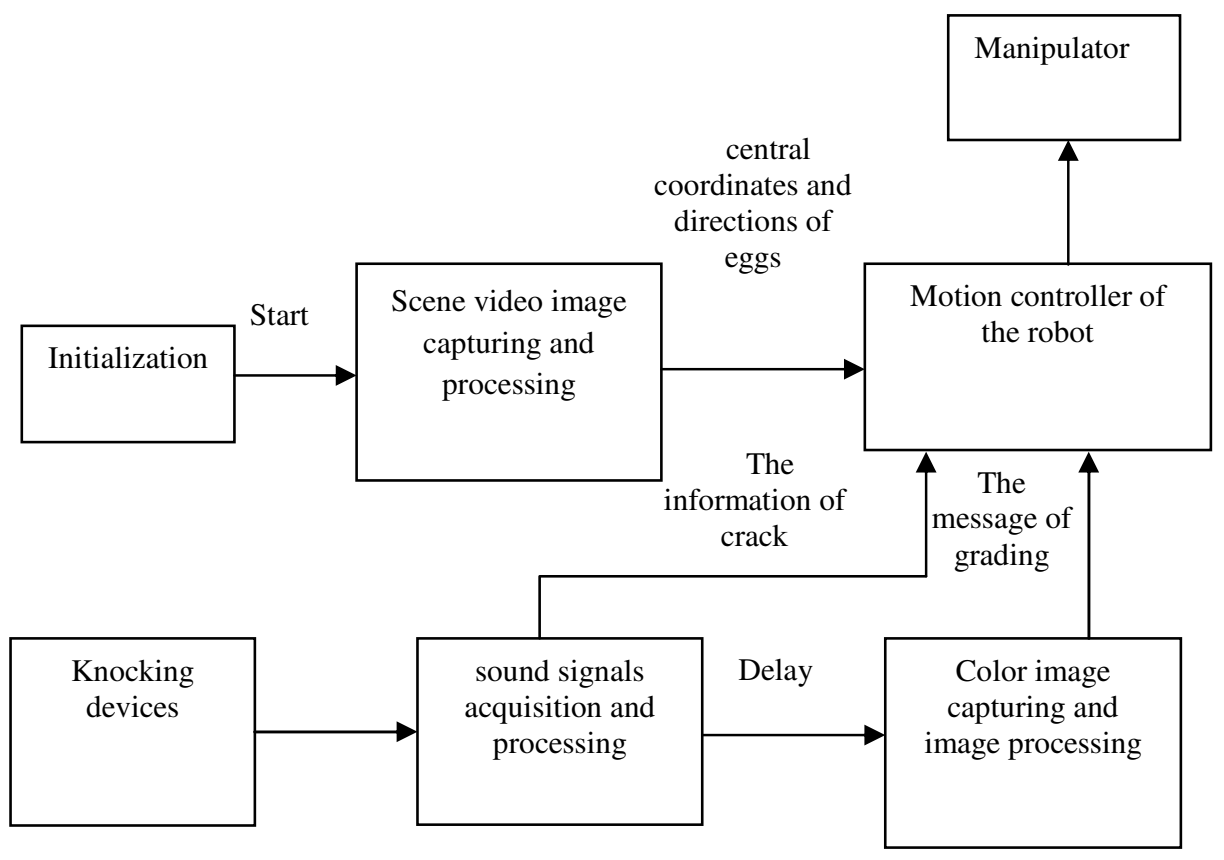

Fig. 1. The working flow of SIRDG

and graded by a corresponding recognition model. Finally, when the grading information is sent to the robot motion controller, the manipulator puts the egg in the corresponding eggcrate.

\section{The Hardware Components of SIRDGE}

\subsection{Video Image Capturing Equipment}

The video image capturing equipment consists of CCD camera, image acquisition card, image display card, controlling computer and so on. Black and white CCD camera image processor is $\mathrm{LG} 1 / 3^{\prime \prime} \mathrm{CCD}$, the system signal PAL standard pattern, the sensitization area $4.9 \mathrm{~mm} \times 3.7 \mathrm{~mm}$, the scanning frequency $15.625 \mathrm{kHz}$ (horizontal) and $50 \mathrm{~Hz}$ (vertical), and the pixel $500 \times 582$. The image acquisition card, DK-2000 Video Capturing Card by Tomoos Inc., is based on PCI bus, also compatible with windows PNP (Plug and Play), of which maximum display resolution is $640 \times 480,24$ bit color, sampling frequency 14.7 MHz.

\subsection{The Robot Reality-The Manipulator}

The study adopts a Japan-made precise universal robot called Move Master-EX which has a light joint opened-chain linkage with 5-DOF (5-degree-of-freedom). 
The main technical parameters of Move Master-EX are as follows: the maximum speed is $1.23 \mathrm{~m} / \mathrm{s}$, the maximum load on wrist joint $1.00 \mathrm{~kg}$, repeated positioning accuracy $0.05 \mathrm{~mm}$, moving scope $(\theta 1, \theta 2, \theta 3, \theta 4, \theta 5)=\left(300^{\circ}, 130^{\circ}, 110^{\circ}, 180^{\circ}, 360^{\circ}\right)$.

\subsection{Robot Motion Controller}

The robot motion controller of Move Master-EX consists of motion controller, IBM-PC or its compatible computer, servo motor with incremental encoder on the robot reality, driver, driver power supply of the driver, $+12 \mathrm{~V} \sim+24 \mathrm{~V} \mathrm{DC}$ supply (for interface card power supply), original point switch, positive/negative limit switch and so on. Move Master-EX uses AC servo motor and has multiple ways of motion control. The system adopts Open-architecture PMAC Motion Controller by Delta Inc., in which each clip can synchro-control 8 32 motion axes and parallel them to achieve multiple axes coordinate motion. The core of PMAC is composed of Motorola DSP56K Digital Signal Processor and FPGA to achieve control calculation of high performance. The system adopts PMAC Motion Controller of PCI Switchboard, which provides RS232 serial communication port, language $\mathrm{C}$ math library and Windows DLL(dynamic-link library). Such application modules as data processing required by control math and system, interface display, and users interface are integrated to construct the control system that meets the requirement of poultry eggs detection and grading.

\subsection{Robot End Effector-Vacuum Sucker}

The original end effector of Move Master-EX Robot is two semi-circular chucks. Because the conveying subjects of the study are extremely friable poultry eggs, the original end effector of Move Master-EX is replaced with vacuum sucker made from silicone rubbers. Experiment shows that the reliable, soft and controllable sucker is good at sucking and discharging poultry eggs.

The gas circuit control of the vacuum sucker adopts AT89S52 MCU, which corresponds with the host computer via MAX232, uses interruption program, sends processing result from pin 2 and 3 of AT89S52 MCU (P1.1and P1.2) to two 4N25 optocouplers. If P1.1 or P1.2 sends low level, the connected optocoupler flows, the coil of DS2Y SSR (Solid State Relay) energizes, the main contact of SSR switches on, the controlled electromagnetic valve energizes, the gas circuit flows and the eggs can be sucked up or put down.

\subsection{Crack Detection Device}

When the eggs are sucked by the sucker and moved along with the manipulator to crack detection point, they shadow the lights of the optoelectronic sensor. Then the optoelectronic sensor produces a voltage signal. The signal is sent through current processing circuit to the interrupt interface of MCU (AT89C51). The MCU accepts this interrupt application, sends pulse signal, drives stepper motor via driving circuit, which further drives the knocking stick to knock on the eggshell. This device can fulfill multipoint knocking and knocking force control. The knocked eggshell produces sound signal, which is converted into electric signal via sound sensor (microphone). The sound signal enters the interrupt interface of DSP (TMS320VC33) after being 
amplified, filtered and getting through threshold trigger circuit. The DSP accepts this interrupt signal and starts A/D converters, which convert the filtered signal into digital signal by sampling, perpetuating, quantization and coding.

\subsection{Inner Quality Detection Equipment}

Inner quality detection hardware consists of illumination, color camera, image acquisition card and computer. The system uses Beijing Daheng DH-VRT-CG200 Image Acquisition Card (Parameters: video source illumination is 20, contrast 20, gray 4 , hue 18 , saturation 50, image sharpness 3, resolution $640 \times 480$, pixel depth RGB24) to convert the video signal from CCD into digital signal that can be recognized by computer, sending the signal via computer PCI bus to computer inner storage of video signal by DMA controller so as to complete the external image acquisition by the computer.

\section{Software Algorithm of SIRDGE}

The main program of SIRDGE software is made up of 5 molds. The module of scene image capturing and processing, the module of motion controller of the robot, the module connected to AT89S52, the module communicated with DSP and the mode of color image capturing and processing. The control and crack recognition of the vacuum sucker are accomplished respectively by AT89S52 MCU and DSP hardware.

\subsection{Video Image Capturing and Camera Calibration}

The flow of the image acquisition card in image capturing is as follows: beginning (initialization), parameter setting, acquired images to the screen or memory, and closing (resource releasing). First the disc installation program along with the image acquisition card is run before programming and the needed library DSStream.DLL is obtained. In the program the relevant include file (.h) is used, and Static Linking for Library (.lib) is added to project file to be used by Compiler Monolithic in linking. The function library file offered by the image acquisition card is added to VC program, and before the image acquisition system works properly, the appointed image acquisition card must be opened first. The last parameter setting before closing is read out from initialization file for initializing, the needed system setting is obtained, and quotation handle of the card is constructed to be quoted by various functions.

This study employs the method of linear camera calibration, which enables the central pixel coordinates of the eggs in the images to correspond with the absolute coordinates of the eggs' center in reality under the robot coordinates through the conversion from image pixel coordinates to image plane coordinates, from image plane coordinates to camera coordinates and from camera coordinates to robot coordinates (absolute coordinates).

\subsection{Image Processing and Feature Extraction}

Gray images acquired by the acquisition card is greatly influenced by lights. When several eggs are close to each other, the division between eggs is difficult to be 
distinguished in the images. Furthermore, image noises also have an effect on sequent positioning accuracy. As a result, the acquired gray images must be processed, including gray threshold transform, image denoising, image dilation and erosion, image connected components label as well as feature extraction of eggs' central coordinates and maximum axis direction.

From the gray histogram of several images in the experiment it can be seen that double peaks appear in the histogram, in which the host peak (255) is the egg while the subordinate peak (about 50) is the background. The bottom of the valleys is between 120 and 250. Image threshold transform is convenient for sequent processing. Adopting the transform function as follows

$$
f(x)=\left\{\begin{array}{cc}
0 & x<250 \\
255 & x \geq 250
\end{array}\right.
$$

After image threshold transform, the image becomes black and white image.

From the optimal gray threshold transform, area of the egg and background areas can be clearly distinguished. However, tiny glistening spots (dirt, paper scraps, water stain, etc.) appear to be white like the egg area. Moreover, image noises make small white dots appear in the background areas and small black dots in the egg area. In order to eliminate noises and obtain ideal images, this study applies Gauss Function of 2D mean value discretization to image smoothing.

Owing to the existence of connecting on the edge between eggs in the images, erosion is applied to the images in order to detach all close eggs. The function of erosion in mathematical morphological calculation is to eliminate boundary points of an object. With white dots of the structure element [3]×[3], an egg image processed above is eroded and a pixel will be lost on the egg edge. When there is fine connecting between two egg images, two eggs can be detached as long as the structure element is big enough.

While programming, image erosion calculation is fulfilled by a function named ErosionDIB () . ErosionDIB () has several parameters: LPSTR lpDIBBits (the pointer pointing to original image), LONG lWidth(the width of the original image), LONG lHeight(the height of the original image), int nMode (the mode of erosion) and int structure [3][3]( structure element).

After the image is eroded, the size information of the egg area is lost. For egg size reverting, the image can be dilated again with the same structure element. It has been found in the experiment that the result of direct dilation is image reverting and egg reunion. The reason is that closed angles have been left in the original joint in the eroded image, which are also dilated in dilation and result in egg reunion. Cross use of smoothing and threshold transform is a feasible method to eliminate closed angles. Therefore, the study first smoothes and threshold transforms the eroded image, and then dilates the image with the structure element the same as erosion.

In order to extract the location coordinates and angle of each egg for the robot to snatch it precisely, each egg must be labeled. Before labeling the egg areas have been distinguished by all the above mentioned means. Therefore, labeling each egg is to label the connected components in the image.

Locating connected components in an image is one of the commonest calculations in machine vision. Dot composition in connecting area indicates optional area. 
Connecting label algorithm can locate all the connected components in the image and distribute the same label to all the dots in one connected component. The study employs four connected components sequential algorithm to label each egg areas.

After the eggs are labeled, the features of the location coordinates and azimuth angles of each egg in the image can be extracted. The ratio of first-order matrix and total pixel area serves as the coordinates of each egg's center under the image coordinates. For a binary image with $\mathrm{n}$ lines and $\mathrm{m}$ columns, pixel point in Line $\mathrm{i}$ and Column $\mathrm{j}$ is $\mathrm{B}(\mathrm{i}, \mathrm{j})$, and the labeled area is

$$
A=\sum_{i=0}^{n-1} \sum_{j=0}^{m-1} B(i, j)
$$

And the central coordinates of eggs in the image coordinates are:

$$
\begin{aligned}
& - \\
& x=\frac{\sum_{i=0}^{n-1} \sum_{j=0}^{m-1} j B(i, j)}{A} \\
& - \\
& y=-\frac{\sum_{i=0}^{n-1} \sum_{j=0}^{m-1} i B(i, j)}{A}
\end{aligned}
$$

Because an egg is approximately oval, its major axis is defined as the direction of the egg and the angle between normal line of the major axis and Axis $\mathrm{X}$ is defined as the direction angle of the egg. Minimal second-order moment axis sharing the same direction with axes of minimal moment of inertia on 2D plane is defined as major axis for the long objects in the image. The second-order moment axis of an image object is such a line: the distance sum of square from all its points to the line is minimal. The distance square $r_{i j}^{2}$ from pixel points to the line is calculated for the binary image $B(i, j)$ of each egg's labeled area. In order to avoid numerical ill-conditioned problem when the line is approximately vertical, the line is expressed as polar coordinates $\rho=x \cos \theta+y \sin \theta$. In this formula $\theta$ is the angle between the normal line of the major axis and Axis $X, \rho$ is the distance from the line to the origin, as shown in Fig 2. The distance sum of square from all the egg's points to the line is minimal.

The function of the distance sum of square from all the egg's points to the line is

$$
\begin{aligned}
& x^{2}=\sum_{i=0}^{n-1} \sum_{j=0}^{m-1} r_{i j}^{2} B(i, j) \\
& r_{i j}{ }^{2}=(i \cos \theta+j \sin \theta-\rho)^{2}
\end{aligned}
$$

After seeking extremum, $\theta$ and $\rho$ can be worked out so as to determine the direction of the egg. 


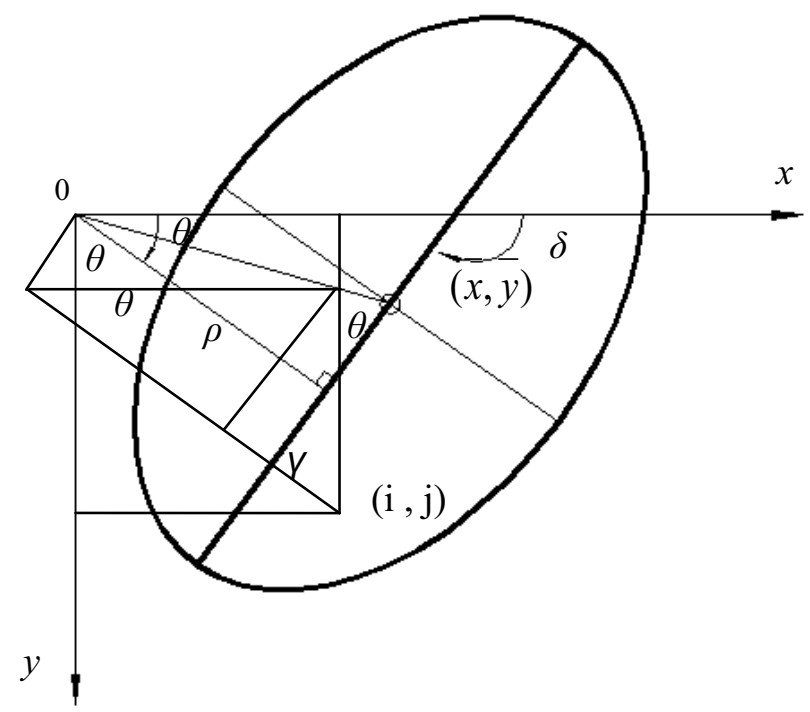

Fig. 2. The definition of the direction of the egg

\subsection{Robot Motion Control and Route Planning}

The robot manipulator motion in picking up eggs can be decided by pose of manipulator nodes sequencing. Each node is described by homogeneous transformation of sucker center coordinates (tool coordinate system) relative to workpiece coordinate system. The corresponding joint variable can be calculated by Kinematics Converse Solution program.

According to the origin of workpieces and major axis direction of the eggs in workpiece coordinate system worked out by the host computer in image processing system. The transforms coordinates is ${ }_{T}^{B} T={ }_{S}^{B} T{ }_{G}^{S} T{ }_{T}^{G} T$. In this formula ${ }_{T}^{B} T$ is the transform from tool coordinate system to spacecraft-referenced coordinates, ${ }_{S}^{B} T$ is the transform from reference coordinates to spacecraft-referenced coordinates, ${ }_{G}^{S} T$ is the transform from workpiece coordinate system to reference coordinates, and ${ }_{T}^{G} T$ is the transform from tool coordinate system to workpiece coordinate system. Hence the host computer obtains coordinate transformation matrix of the robot end effector (sucker) from the current point to the next one. Then the host computer obtains rotation angles of all the robot's joints $\theta 1 \sim \theta 5$ by means of robot kinematics converse solution, and gives corresponding control orders to motion control card according to the given acceleration and top speed. According to the control orders the motion control card accelerates the electromotor of every joint of the robot at the given acceleration to top speed or starts to decelerate until it stops at the set place. The sensor sends the position signal to the host computer, which gives orders to MCU of the sucker pneumatic system after receiving the signal. The MCU controls electromagnetic valve to switch on 
the gas path and suck up or put down the eggs. In order to fully accomplish detection and grading in a process of sucking up and putting down an egg, the planning is carried out to the robot motion path. The complex course in which jumping-off point and terminal are both variational in detection and grading is simplified into three easily fulfilled steps: the jumping-off point is variational but the terminal immovable (from sucking the egg to crack detection position); the jumping-off point and terminal are both immovable (from crack detection position to quality defect detection position); the jumping-off point is immovable but the terminal variational (from quality defect detection position to the corresponding eggcrate the egg is carried into). Such treatment enables the robot motion control to be realized easily.

In order to make use of the dynamic link libraries, Pmacu.h, m_hPmac.lib and PmacPc.dll (in the path of Windows $\backslash$ Dll of the disc attached to the product ) ,of motion control card , \#include "pmacu.h" is first added to the program, then project-setting-link is chosen in VC menu, then m_hPmac.lib is entered in object/library modules, and finally the function in dynamic link libraries of motion control card can be used in the program.

\section{Property Testing of SIRDGE}

SIRDGE is composed of the above software. The system gives the scene image gray threshold transform, smoothing using Gauss template, image dilation and erosion, image connected components label, the result of which is shown in Fig 3.

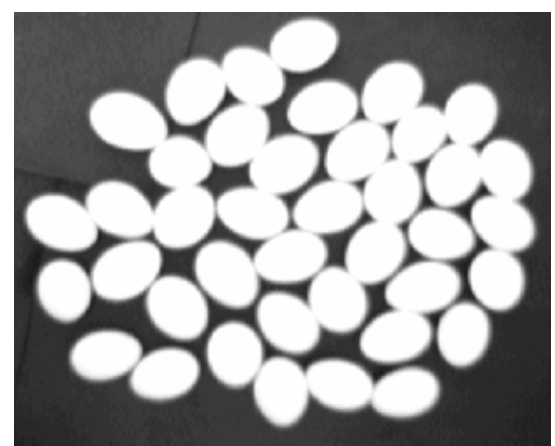

Before image processing

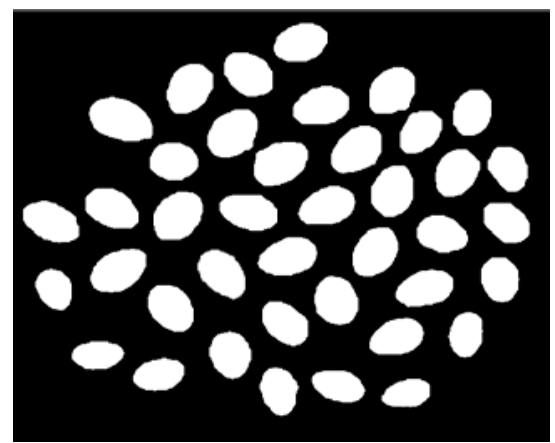

After image processing

Fig. 3. Comparison of pre-and post-image processing

In order to extract the absolute coordinates of the eggs' center, nine calibration points are used to calibrate the scene camera. Then nonlinear regression models are used to obtain the conversion matrix parameter from the central coordinates of the eggs in the images to the absolute coordinates of the eggs' center in reality space.

The rotation matrix is

$$
R=\left[\begin{array}{ccc}
0.02 & -1.08 & 0.14 \\
1.25 & 0.05 & 0.08 \\
0.12 & 1.03 & 1.16
\end{array}\right]
$$


The shift matrix is

$$
T=\left[\begin{array}{c}
-352 \\
447 \\
598
\end{array}\right]
$$

The robot motion experiment has been conducted in Intelligent Robot Institute, Central China University of Science and Technology. Fig 4 is the experiment photo.

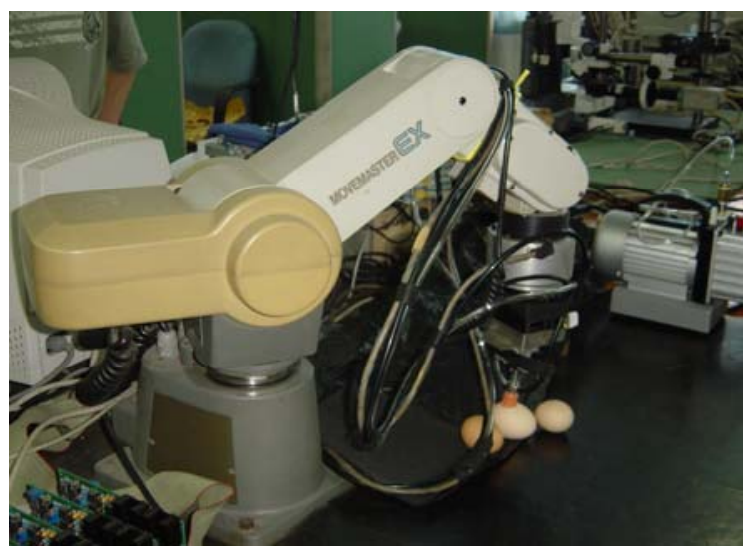

Fig. 4. The picture in experiment

\section{Conclusion}

SIRDGE is able to automate fully egg detection and gradation, apply systematically and comprehensively the technology on computer vision, image processing, robot motion control, sound detection and negative pressure, and has changed the situation that huge operation mechanism or manual charging/discharging is needed in the existing egg detection and gradation machinery.

The vacuum sucker serves as robot end effector. The designed sucker, vacuum suction gas path and MCU have good effects in conveying, sucking and discharging poultry eggs.

The ratio of first-order matrix of digital image and total pixel area serves as the coordinates of each egg's center under the image coordinates. The method of linear camera calibration is employed. Minimal second-order moment axis sharing the same direction with axes of minimal moment of inertia on 2D plane is defined as maximum axis for the egg. This study applies optimal threshold transform and Gauss Function of 2D mean value discretization to image smoothing, uses several times of image dilation and erosion, makes image segmentation, and employs four connected components sequential algorithm to label the same egg area in the image. The corresponding algorithm obtained can meet the working demand for leading the robot to locate each egg precisely. 
By means of robot kinematics converse solution, the absolute coordinates of the eggs' center and maximum axis direction are transformed into rotation angles of all the robot's joints. In order to fully accomplish detection and grading in a process of conveying, the planning is carried out to the robot motion path. The complex course in which jumping-off point and terminal are both variational in detection and grading is simplified into three steps: the jumping-off point is variational but the terminal immovable; the jumping-off point and terminal are both immovable; the jumping-off point is immovable but the terminal variational. Such treatment enables the robot motion control to be realized easily.

\section{Acknowledgements}

Funding for this research was provided by Chinese National Programs for High Technology Research and Development (project number: 2007AA10Z214). The first author is grateful to professor Xinhan Huang, the Intelligent Robot Institute and the Central China University of Science and Technology for providing him with a Japan-made precise universal robot called Move Master-EX. to do experiments.

\section{References}

[1] Hot Cheers Ind. Inc. Full automatic egg-graders [EB/OL] (2005-05-16), http: / / hotcheers.diytrade.com [2006-11-25]

[2] Elster, R.T., Goodurm, J.W.: Detecting of cracks in eggs using machine vision. Transactions of the ASAE 34(1), 307-312 (1991)

[3] Goodurm, J.W., Elster, R.T.: Machine vision for cracks detection in rotation eggs. Transactions of the ASAE 35(4), 1323-1328 (1992)

[4] Cho, H.K., Won, Y.K.: Crack detection in eggs by machine vision. In: 6th Int. Conf. on Computers in Agriculture, May 15-16, pp. 777-784. St. Joseph. Mich., ASAE (1996)

[5] Nakano, K., Motonaga, Y., Mizutani, J.: Development of non-destructive detector for abnormal eggs. In: Workshop on Control Applications in Post-Harvest and Processing Technology, vol. (1), pp. 71-76 (2001)

[6] Kuchida, K., Fukaya, M.: Nondestructive prediction method for yolk: albumen ratio in chicken eggs by computer image analysis. Poultry Science 78(6), 909-913 (1999)

[7] Das, K., Evans, M.D.: Detecting fertility of hatching eggs using machine vision. Transactions of the ASAE 35(6), 2035-2041 (1992)

[8] Sinha, D.N., Johnston, W.K., Grace, C.L.: Acoustic resonance in chicken eggs. Biotechnology Prog. 8(3), 240-243 (1993)

[9] Ketelaere, E.B.D., Coucke, P., Baerdemaeker, J.D.: Eggshell crack detection based on acoustic resonance frequency analysis. Agricultural Engineering Res. 76(2), 157-163 (2000)

[10] Shucai, W., Yilin, R., Hong, C., Lirong, X., Youxian, W.: Detection of cracked-shell eggs using acoustic signal and fuzzy recognition. Transactions of The Chinese Society of Agricultural Engineering 21(4), 130-133 (2004)

[11] Jianying, L., Jiayan, C., Youchun, D., Yilin, R., Shucai, W., Lirong, X., Dongjiao, C., Youxian, W.: Model for automatic detection of eggshell crack. Transactions of The Chinese Society of Agricultural Engineering 21(9), 189-192 (2005) 
[12] Lirong, X., Youchun, D., Hong, C., Youxian, W.: Study On the Automatic Detection System of Duck Egg Classification. Journal of Huazhong Agricultural 19(4), 133-137 (2000)

[13] Qiaohua, W., Yilin, R., Youxian, W.: Study on Non-destructive Detection Method for Fresh Degree of Eggs Based on BP Neural Network. Transactions of the Chinese Society for Agricultural Machinery 21(1), 158-162 (2006)

[14] Qiaohua, W., Yousheng, Y., Wangyuan, Z., Xiaowei, C., Jianying, L., Youxian, W.: Research on the Grading Model of Duck Egg Bulk. Transactions of the Chinese Society for Agricultural Machinery 17(6), 202-205 (2001)

[15] Lirong, X., Shucai, W., Yilin, R., Youchun, D.: The Revision of Grade Model on Egg's Weight. Journal of Agricultural Mechanization Research (2), 35-37 (2006)

[16] Shucai, W.: A Study of Autonomous Robot Detecting and Grading Eggs, pp. 75-89. Huazhong Agricultural University, Wuhan (2006)

[17] Minghong, R.: Realizing Image Acquisition with AVICAP.DLL in VC++. Computer Knowledge and Technology (Academic Exchange) (24), 1133-1134 (2007)

[18] Zhenyou, Z., Aijie, X., Tao, L., Shanben, C.: High-speed calibration method for the relationship of the eye-in-hand of robot vision. Optical Technique 30(2), 150-154 (2004)

[19] Yuting, J., Zhongke, Y.: Optimal Template for Image Smooth Denoising. Journal of Transportation Engineering and Information (3), 68-71 (2004)

[20] Yunde, J.: Machine Vision, pp. 196-204. Publishing House of Science and Technology (2004)

[21] Hong, X., Fangwen, R.: Numerical stability and morbidity problem. China Science and Technology Information (11), 322-323 (2006)

[22] Ganmin, T., Zhongke, S.: Measuring and Analyzing Industrial Noise Based on DSP. Microprocessors (2), 28-30 (2006)

[23] Meihu, M., Jiansheng, X., Farong, G., Yongchang, Z.: Research of Egg-Vacuum -Sterilization Preservation-Technology (EVSPT). China Poultry (8), 24-26 (2001) 